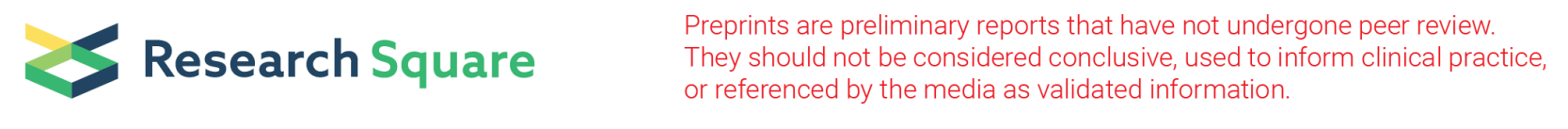

\title{
Can Thoracolumbar Deformity Improve if Surgery Just for Lumbar Stenois Syndrome in Degenerative Thoracolumbar Kyphosis Patients ?
}

\author{
Fanqi Meng \\ Peking University People's Hospital \\ Xianguo Bao \\ Peking University People's Hospital \\ Shuai $\mathrm{Xu}$ \\ Peking University People's Hospital \\ Yan Liang \\ Peking University People's Hospital \\ Haiying Liu ( $\square$ liuhaiying1131@sina.com ) \\ Peking University People's Hospital
}

\section{Research article}

Keywords: degenerative thoracolumbar kyphosis, lumbar spinal stenois syndrome, short-level fixation, spino-pelvic alignment, influencing factors

Posted Date: August 21st, 2020

DOI: https://doi.org/10.21203/rs.3.rs-58696/v1

License: (c) (i) This work is licensed under a Creative Commons Attribution 4.0 International License. Read Full License 


\section{Abstract}

Background: Fusion across thoracolumbar spine or not for degenerative thoracolumbar kyphosis (DTLK) in lumbar stenois syndrome (LSS) remains controversial. The influencing factors for postoperative TLK in this group have not been determined yet. So the study was to explore whether DTLK could improve with only surgery for lumbar stenois syndrome LSS and identify influencing factors on postoperative TLK.

Methods: The study was performed from January 2016 to December 2018. 69 participants (25 male) diagnosed LSS with DTLK were enrolled and surgery was only for LSS. Radiological parameters included TLK, lumbar lordosis (LL), pelvic incidence (PI), pelvic tilt (PT), sacral slope (SS) and osteoporosis. Clinical outcomes were visual analogue scale (VAS) and Oswestry disability index (ODI). According to lower instrumented vertebrae (LIV) on L5 or S1, inter-group comparisons were performed between LIV on L5 (L5 group) and S1 (S1 group).

Results: Demographics was well-matched between L5 and S1 group with a mean follow-up of $24.3 \pm 12.1(\mathrm{~m})$. TLK improved with a mean of $16.2 \pm 7.6\left(^{\circ}\right)(\mathrm{P}<0.001)$. It was insignificant on radiological and clinical parameters between L5 and S1 groups except a larger PT in S1 group $(P=0.046)$. VAS $(P=0.787)$ and ODI $(P=0.530)$ were both indifference between normal TLK and DTLK at last $(P \llbracket 0.05)$. Postoperative TLK was affected by osteoporosis and SS, the latter was determined by PI and PT. Osteoporosis was the risk factor for TLK correction ( $P=0.001$, $\mathrm{OR}=9.58)$.

Conclusions: DTLK get improved if suegery only performed for LSS. TLK and clinical outcomes are comparable between L5 and S1 groups. Severe osteoporosis can impede TLK correction.

\section{Background}

Degenerative thoracolumbar kyphosis (DTLK), as a kind of adult spinal deformity, is a common degenerative spinal disease in the elderly [(1)]. Lumbar spinal stenois syndrome (LSS) combined with DTLK, with gradually increassing exposure rate nowadays, can results to low back pain and lower extremities dysfunction [(2)], which is sometimes cured by surgery, and the majority of the series mind the symptom of LSS instead of DTLK [(3)]. There is no doubt of operating on responsible levels with severe LSS, disc protrusion or instability, while whether instruments implanting and deformity correction acrossing or referring to the region of TLK has been filling with controversary $[(4,5)]$.

It is reported numerous successes hava been achieved on TLK deformity through 1 to 3 grade osteotomy and long-segment instrument spanning across this region, while adverse outcomes have emerged such as extensive trauma and operating duration, huge expenses and even instrument-related infection $[(6,7)]$. Recently, more and more experienced surgeons proposed the defination of short-segment fixation and the theory of precision therapy $[(8,9)]$, by which it was enough to desolve the chief clinical complaints rather than correcting all malformation into a resonable range. Shin et al. [(10)]suggested simple decompression surgery, not the disordered levels, was able to restore satisfied sagittal alignment in $70 \%$ of patients caused by LSS, but the point was not further identified on LSS combined with DTLK yet.

There is biomechanical interaction among focal sagittal parameters of spino-pelvic alignment, where thoracolumbar segments, as a bridge element, is probably affected by other regional parameters $[(1,11)]$. Once $\mathrm{S} 1$ was chosen as lower instrumented vertebrae (LIV), the sacral slope (SS) and pelvic tilt (PT) was hardly to be changed since the pelvic incidence (PI) was almost fixed in the adult where PI=PT+SS. So, the parameters may be significant when L5 was as LIV with more range of pevic rotation $[(12,13)]$. While it is unclear whether TLK can be influenced when L5 or S1 is chosen as LIV in short-segment fusion through biomechanical chain.

Therefore, the study included patients diagnosed as LSS combined with DTLK, who were performed short-segment fixation simply for LSS, where upper instrumented vertebrea (UIV) was lower than L2. A short-term follow-up was completed to identify (1) whether DTLK could improve without intervention to thoracolumbar region, (2) whether TLK was different with LIV on L5 or S1 and (3) the influenced factors on TLK after surgery.

\section{Methods}

\section{Patients enrollment}

The single-center retrospective protocal was performed from January 2016 to December 2018. The participants diagnosed LSS combined with DTLK was enrolled. The study was approved by local institutional review board and all patients have signed informed consent.

The inclusion criteria included (1) the patients were larger than 55 years, (2) patients were diagnosed as LSS with surgery indication, (3) patients suffered from sagittal TLK resulted difinitely from degeneration (TLK $\left.\geq 15^{\circ}\right)$, (4) local fixation for LSS: UIV was not above L2 and LIV was L5 or S1, (5) intact stand lumbar and whole spine X-ray and lumbosacral MRI could be obtained, (6) it was primary surgery and (7) without coronal deformity. The exclusion criteria were (1) LSS not reached surgical-indication yet, (2) TLK dued to non-degenerated deformity 
such as ankylosing spondylitis, compressed facture or Scheuermann's disease, (3) with coronal deformity, (4) inner-fixation referred to or stretched accross thoracolumbar levels, (5) incomplete or unclear radiological data for measurement, (6) secondary or revised surgery and (7) loss of follow up.

For DTLK cases, posterior lumbar interbody fusion (PLIF) and posterior-lateral fusion (PLF) was applied mainly for lumbar segments with spinal stenosis, disc hernia and instability, which was instrumented in-situ or with grade 1 to 3 osteotomy. All patients was operated by one senior surgeon.

\section{Radiological parameters and clinical outcomes}

Radiological parameters included kyphosis apex, thoracic kyphosis (TK), TLK, lumbar lordosis (LL), PI, PT and SS. TK was the angle between upper endplate of T5 and lower endplate of T12; TLK was the angle between upper endplate of T10 and lower endplate of L2; LL was between upper endplate of L1 and upper endplate of S1. The defination for PI, PT and SS was shown in Fig 1 (Fig 1). Osteoporosis was determined by X-ray, MRI and surgical records, where severe osteoporosis was manifested by decreased bone density, thinned trabecular bone, biconcave or wedge-shaped changes in vertebrae, which was together evaluated during operation[(14)].

Clinical outcomes were evaluated by visual analogue scale (VAS) and Oswestry disability index (ODI). VAS ranged from 0 to 10 and a higher score implied more severe pain. ODI reflected disability on lumbar spine function and quality of life (0-50 score) and higher index represented more disability. All outcomes were measured before operation (baseline) and at follow-up endpoint.

Furthermore, according to LIV on L5 or S1, inter-group comparisons were performed between LIV on L5 (L5 group) and S1 (S1 group).

\section{Statistical analysis}

The measurement data was depicted as mean \pm standard deviation. The dichotomous between groups were analyzed by $\chi^{2}$ test. Independent sample t test was used for inter-group measurement data and Paired t test was applied between baseline and endpoint, while Wilcoxon test was used for ordinal data. Pearson or Spearman correlation analysis were used among parameters and between TLK and demographics. Multiple linear regression and logistic regression were for determining influencing factors of TLK. SPSS 22.0 (IBMC, Armonk, New York, USA) the software for statistical analysis and $\mathrm{P}<0.05$ was significant difference.

\section{Results}

A total of 69 DTLK patients (25 male) was included with a mean follow-up of $24.3 \pm 12.1(\mathrm{~m})$, the age and body mass index (BMI) were respectively $68.9 \pm 9.2(55-84)(y)$ and $26.1 \pm 3.5\left(\mathrm{~kg} / \mathrm{m}^{2}\right)$. The most operated segments was L2-L5 (29.0\%), followed by L3-L5 (24.6\%) and L3-S1 (14.5\%). There were no significances in gender, age and BMI between L5 group and S1 group, so was osteoporosis (P $\otimes 0.05)$. The number of operated segments was larger in S1 group $(P<0.05)$ (Table 1).

Table 1

Basic information between L5 group and S1 group of DTLK

\begin{tabular}{|llll|}
\hline Statistics & L5 group & S1 group & P \\
\hline Gender(M: F) & $17: 27$ & $8: 17$ & 0.581 \\
\hline Age $(\mathrm{y})$ & $69.1 \pm 8.7$ & $67.5 \pm 10.2$ & 0.494 \\
\hline BMI $\left(\mathrm{kg} / \mathrm{m}^{2}\right)$ & $25.9 \pm 3.2$ & $26.6 \pm 4.0$ & 0.422 \\
\hline Osteoporosis & 21 & 12 & 0.983 \\
\hline No. of screws & $6.5 \pm 1.5$ & $7.7 \pm 1.7$ & 0.006 \\
\hline No. of segments & $2.3 \pm 0.8$ & $2.8 \pm 0.9$ & 0.006 \\
\hline Follow up (m) & $24.2 \pm 12.1$ & $24.6 \pm 12.5$ & 0.899 \\
\hline Footnote: DTLK: degenerative thoracolumbar kyphosis; M: male; F: female
\end{tabular}

At the endpoint, 63 cases (91.3\%) got decrease on TLK by a mean of $8.9 \pm 11.5\left(^{\circ}\right)$ and 27 patients acquired normal TLK. Thus, TLK improved with a mean of $16.2 \pm 7.6\left(^{\circ}\right)$ compared to baseline $(P<0.001)$, so did TK $(P=0.022)$. While there were no statistical differences on $L L$, $P I$, $P T$ and 
SS between the two time points $(\mathrm{P} \otimes 0.05)$. In addition, VAS and ODI both decreased $(1.9 \pm 1.6$ and $7.9 \pm 6.3$, respectively) at last (both $P<0.001)$

(Table 2). At baseline, there were no significances on radiological parameters between L5 and S1 group, so were VAS and ODI ( $<0.05)$. These parameters were also comparable at the endpoint between groups except a larger PT (22.5 \pm 8.6$)$ in S1 group ( $P=0.046)$. In L5 group, TK

$(20.7 \pm 12.1)$ and TLK $(16.5 \pm 7.3)$ decreased at final compared to baseline $(P=0.041$ and $P=0.001$, respectively) while the others kept stable, the same situation in $\mathrm{S} 1$ group ( $\mathrm{P}=0.047$ and $\mathrm{P}=0.012$, respectively) (Table 3)(Fig 2).

Table 2

Sagittal parameters and clinical outcomes between baseline and endpoint

\begin{tabular}{|llll|}
\hline & Preoperation & Endpoint & P \\
\hline TK $\left(^{\circ}\right)$ & $32.3 \pm 15.8$ & $22.4 \pm 12.5$ & 0.022 \\
\hline TLK $\left(^{\circ}\right)$ & $24.9 \pm 8.3$ & $16.2 \pm 7.6$ & 0.001 \\
\hline LL $\left(^{\circ}\right)$ & $38.3 \pm 18.1$ & $40.1 \pm 14.1$ & 0.217 \\
\hline PI $\left(^{\circ}\right)$ & $46.2 \pm 11.7$ & $46.7 \pm 10.4$ & 0.630 \\
\hline PT $\left(^{\circ}\right)$ & $19.7 \pm 8.1$ & $19.4 \pm 9.8$ & 0.736 \\
\hline SS $\left(^{\circ}\right)$ & $26.6 \pm 10.9$ & $27.3 \pm 9.5$ & 0.527 \\
\hline VAS & $6.6 \pm 1.4$ & $1.9 \pm 1.6$ & $<0.001$ \\
\hline ODI & $35.8 \pm 6.9$ & $7.9 \pm 6.3$ & $<0.001$ \\
\hline Footnote: TK: thoracic kyphosis; TLK: thoracolumbar kyphosis; LL: lumbar lordosis; PI: pelvic incidence; PT: pelvic tilt; SS: sacral slope; & \\
\hline VAS: visual analogue scale; ODI: Oswestry disability index & \\
\hline
\end{tabular}

Table 3

Sagittal parameters and clinical outcomes between L5 and S1 group

\begin{tabular}{|c|c|c|c|c|c|c|}
\hline & \multicolumn{3}{|c|}{ Preoperation } & \multicolumn{3}{|l|}{ Endpoint } \\
\hline & L5 group & S1group & $P$ & L5 group & S1group & $\mathrm{P}$ \\
\hline $\operatorname{TK}\left({ }^{\circ}\right)$ & $31.0 \pm 15.8$ & $34.3 \pm 15.9$ & 0.427 & $20.7 \pm 12.1^{\star}$ & $24.4 \pm 13.8^{*}$ & 0.577 \\
\hline $\operatorname{TLK}\left({ }^{\circ}\right)$ & $25.9 \pm 9.1$ & $23.2 \pm 6.2$ & 0.189 & $16.5 \pm 7.3^{\star \star}$ & $15.8 \pm 8.3^{*}$ & 0.760 \\
\hline $\operatorname{LL}\left({ }^{\circ}\right)$ & $36.8 \pm 16.0$ & $40.7 \pm 20.5$ & 0.386 & $39.6 \pm 14.0$ & $41.0 \pm 14.5$ & 0.691 \\
\hline $\mathrm{PI}\left({ }^{\circ}\right)$ & $45.0 \pm 11.3$ & $47.9 \pm 12.0$ & 0.305 & $46.1 \pm 10.7$ & $47.9 \pm 9.9$ & 0.506 \\
\hline PT $\left({ }^{\circ}\right)$ & $19.2 \pm 7.1$ & $20.5 \pm 9.4$ & 0.453 & $17.7 \pm 10.0$ & $22.5 \pm 8.6$ & 0.046 \\
\hline SS $\left(^{\circ}\right)$ & $26.1 \pm 10.0$ & $27.4 \pm 12.0$ & 0.638 & $28.3 \pm 9.4$ & $25.3 \pm 9.6$ & 0.225 \\
\hline VAS & $6.6 \pm 1.4$ & $6.6 \pm 1.3$ & 0.937 & $1.9 \pm 1.4$ & $1.8 \pm 1.9$ & 0.851 \\
\hline ODI & $35.2 \pm 7.7$ & $37.0 \pm 5.1$ & 0.363 & $7.7 \pm 5.1$ & $8.3 \pm 8.1$ & 0.706 \\
\hline \multicolumn{7}{|c|}{$\begin{array}{l}\text { Footnote: TK: thoracic kyphosis; TLK: thoracolumbar kyphosis; LL: lumbar lordosis; PI: pelvic incidence; PT: pelvic tilt; SS: sacral slope; } \\
\text { VAS: visual analogue scale; ODI: Oswestry disability index }\end{array}$} \\
\hline
\end{tabular}

Many radiological parameters were highly correlated to each other at the endpoint while TLK was just negetively to $\mathrm{PI}(\mathrm{r}=-0.340, \mathrm{P}=0.010)$ and SS ( $r=-0.415, P=0.001)$ (Table 4). There were no relationships between TLK and VAS $(P=0.678)$ or ODI $(P=0.786)$ at last. What's more, VAS $(P=0.787)$ and ODI $(P=0.530)$ were both of no significances between ones with normal TLK and DTLK series at last. TLK was not related to gender $(P=0.973)$, age $(P=0.749)$ and $B M I(P=0.636)$ at last, but positively correlated to osteoporosis $(r=0.343, P=0.009)$. 
Table 4

Correlation analysis between TLK and radiological or clinical parameters at endpoint

\begin{tabular}{|c|c|c|c|c|c|c|c|c|c|c|c|c|c|c|}
\hline & TK & & TLK & & LL & & $\mathrm{PI}$ & & PT & & SS & & VAS & \\
\hline & $r$ & $\mathrm{P}$ & $r$ & $\mathrm{P}$ & $r$ & $\mathrm{P}$ & $r$ & $P$ & $r$ & $\mathrm{P}$ & $r$ & $\mathrm{P}$ & $r$ & $\mathrm{P}$ \\
\hline TLK & -0.206 & 0.445 & & & & & & & & & & & & \\
\hline LL & 0.576 & 0.019 & -0.213 & 0.112 & & & & & & & & & & \\
\hline PI & 0.136 & 0.615 & -0.340 & 0.010 & 0.441 & $<0.001$ & & & & & & & & \\
\hline PT & -0.338 & 0.200 & 0.031 & 0.817 & -0.248 & 0.024 & 0.555 & $<0.001$ & & & & & & \\
\hline SS & 0.364 & 0.166 & -0.415 & 0.001 & 0.732 & $<0.001$ & 0.523 & $<0.001$ & -0.418 & $<0.001$ & & & & \\
\hline VAS & 0.079 & 0.828 & 0.062 & 0.678 & -0.073 & 0.593 & 0.030 & 0.827 & 0.081 & 0.551 & -0.064 & 0.640 & & \\
\hline ODI & 0.175 & 0.630 & -0.040 & 0.786 & -0.044 & 0.746 & 0.135 & 0.320 & 0.216 & 0.110 & -0.096 & 0.481 & 0.848 & $<0.001$ \\
\hline
\end{tabular}

TLK at the endpoint was regarded as depended variable, where parameters with $\mathrm{P}<0.2[(15)]$ in correlation analysis were included as independent variables. Table 5 showed lower SS and severe osteoporosis were risk factors for postoperative TLK (Beta=-0.427, $\mathrm{P}=0.025$ and Beta=0.374, P=0.039) (Table 5). SS was determined by PI and PT, where SS= PI-PT. When postoperative TLK was seen as dichotomous by corrected TLK and DTLK, logistic regression was performed. When LL, PI, SS and severe osteoporosis were pooled, it showed severe osteoporosis was the independent risk factor for TLK correction ( $\left.P=0.001, \chi^{2}=14.97,0 R=9.58,95 \% \mathrm{CI}[2.49,36.93]\right)$.

Table 5

Multiple linear regression analysis of TLK at endpoint

\begin{tabular}{|c|c|c|c|c|c|}
\hline \multirow[t]{2}{*}{ Coefficient } & \multicolumn{2}{|c|}{ Unstandardized } & \multirow{2}{*}{$\begin{array}{l}\text { Standardized } \\
\text { Beta }\end{array}$} & \multirow[t]{2}{*}{$\mathrm{T}$} & \multirow[t]{2}{*}{$\mathrm{P}$} \\
\hline & $\mathrm{B}$ & SE & & & \\
\hline (constant) & 27.336 & 4.561 & & 5.993 & 0.000 \\
\hline LL & 0.111 & 0.095 & 0.202 & 1.158 & 0.252 \\
\hline PI & -0.122 & 0.106 & -0.170 & -1.147 & 0.256 \\
\hline SS & -0.343 & 0.149 & -0.427 & -2.306 & 0.025 \\
\hline Osteoporosis & 2.612 & 1.871 & 0.374 & 2.396 & 0.039 \\
\hline
\end{tabular}

\section{Discussion}

DTLK, as a kind of ASD, has been one of the hot topics in the feld of spine surgery with the incidence of 5\%-34\% [(7)]. The majority of the population have no obvious clinical manifestations with occasionally mild low back pain and paraspinal muscle tension [(1)]. Only a small group visits the doctors with severe restriction on quality of life and sagittal malalignment, let alone the proportion of surgical-demanding. Although increasing requirement on body shape, it may be undserved to perform orthopdics with huge cost, enlarged invasion while lower effectiveness, especially for the middle-aged and elderly [(16)]. Due to the shorter rest-survival expectation, self-regulation or conservative treatment is usually adopted for the most. However, as the most common degenerative spinal disease in this group, LSS can induce lower extremities pain and intermittent claudication with poor quality of life and there is no doubt that surgical treatment on responsible segment is chosen for most cases with severe LSS [(10)]. The population diagnosed LSS combined with DTLK is non-ignorable based on magnanimous LSS cases, where they often wonder whether DTLK needs to be corrected since the chief complaints are mainly caused by LSS. It is also too confused for many spinal surgeons to give the identified answer.

Surgical correction with osteotomy and long-segment fusion on TLK has proven to be effective, leading to superior body appearance, clinical and radiographic outcomes, especially proper alignment is restored $[(7,17)]$. However, surgical treatment spanning across malformed TLK remains challenging as demonstrated by revisions (9.0-17.6\%) and adverse events such as extensive tissue stripping, burden expenses and rod fracture $[(18,19)]$. With the concept of minimum of invasion and maximum of efficacy, the superiority of precision therapy and short-level

Page 5/11 
instrument are promoting, particularly with more profound knowledge of ASD, qualified implants with better biocompatibility and Young's modulus, improved technology and perioperative management [(20)]. Once lower complications ratio and effective quality of life is still achieved with decompression and fixation only on responsible level, it will be significant in saving marvelous wealth for society, government and themselves. This study was retrospectively performed on cases with LSS and DTLK, where the series underwent PLIF or PIF only for LSS, not referring to TLK. In total, it showed effective outcomes although there were 4 patients (5.8\%) in instability on upper adjacent segment and 2 cases (2.9\%) in proximal junctional kyphosis while with no symptom at endpoint (data not shown), which proved the concept of "precison fixation" for DTLK ones was appropriate.

Patients with LSS appear with lumbar extension and improve with trunk fexion, where they can take severely stooped posture aiming pain relief [(18)]. Fuji et al. [(21)] observed decompression and proper fixation in LSS was followed by improvement in alignment parameters such as reduction of PT and TK and increase of LL, the same point of Shin et al. [(10)] According to Redaelli's point [(18)], two different categories on TLK was divided: structural and non-structural TLK, the latter acted like inducing adaptation on alignment with reversibility, which was considered compensatory TLK due to focal pathologies. LSS can behave as a focal cause of DTLK, but the way to distinguish the two subsets categories is not clear [(22)]. In our data, TLK ranged from $15.2^{\circ}$ to $45.5^{\circ}$ at baseline and most was regarded as non-structural TLK by radiological measurement and intraoperative judgement.

This study observed DTLK cases acquired improvement on TLK with only treatment for LSS with a mean TLK reduction by about $10^{\circ}$ and normal TLK achieved near to 40\%. On the one hand, adequate decompression of LSS removed pain derived from nerve root compression and ensuing reduced the trunk anteversion [(23)]. On the other hand, the recovery and stability of LL optimized compensation especially on proximal TK and TLK with the motion preserving [(10)]. In addition, the paraspinal muscles got stronger by cooperating with functional exercise after surgery, which probably reduced TLK [(24)].

Sagittal alignment, biomechanism and clinical outcomes has been discussed when L5 or S1 was respectively chosen as LIV. Yasuda et al. [(25)] found fusion to L5 was conducted for selected ASD patients with better ODI and less complex deformity contrasted with S1 as LIV. Choi et al. [(13)] identified S1 double screws are a viable option for sacropelvic fixation in ASD patients when L5 pedicle screw fixation was not possible, and S1 group achieved better reconstruction on LL while restricted pelvis rotation. In this study, the only difference between L5 and S1 group was whether L5-S1 was etiological segment. The clinical outcomes were comparable in L5 and S1 at endpoint but the pelvic retroversion compared to L5 group, which was not consistent to Yasuda et al. while agreed with Choi et al. Although the restrictied motion of L5-S1 in S1 group, the compensary of proximal segment played an important role, which made comparable results on alignments between the 2 groups [(26)]. In addition, both groups acquired decreased TLK and TK at last, mainly because the removal of compression and enlargement of lumbar canal, consequently the stooped posture for pain-relief was corrected $[(2,17)]$, which was in line with Fuji et al.

There are interaction among alignment parameters but TLK seems like estranged from others. It is considered that the abnormality of the transition site is affected by many aspects. Thoracolumbar region locates in the transitional part of both anatomical and biomechanical structure with large shear force, where vertebrae or intervertebral discs involved in the region are prone to wedge-shaped. Then, fibrosis of the anteriot ligament force it closer between adjacent vertebrae, especially in the elderly with heavy work [(27)]. TLK was affected by SS, which was determined by PI and PT. The pelvis is critical factor for sagittal alignment and is responsible for retroversion. Based on the relation SS = PI-PT, the amount of pelvic rotation can even approximate PI with sacral endplate horizontal, so individuals with high PI have wider range of adaptation. This movement is very significant, corresponding to an increase of PT correlated to back pain and disability [(28, 29)].Therefore, the retroversion of pelvis, in association with proximal spine forward leaning and even dorsal hin and knee fexion, analogue to sitting and resulting to capacity over-consumption. However, no relevance between TLK and clinical outcomes suggests that TLK puts little impact on qualitt of life in short-term and adequate decompression on LSS is fundamental method.

Age-related osteoporosis, with sparse trabecular and fragile cortex, can induce thoracolumbar spine deformities, resulting in kyphosis, shortened budy length and trunk anteversion, especially based on dorsally mismatched elastic modulus cause by instrument. Yagi et al. elucidated the role of bone strength for developing proximal junctional kyphosis in 113 ASD with 2-year follow-up, they found osteoporosis was a significant risk factor for kyphosis $(\mathrm{OR}=6.4)$ and suggested surgeons should consider prophylactic treatments when correcting ASD with low bone mineral density [(30)]. In this study, the patients with severe osteoporosis was as almost 10 times as patients without osteoporosis in sufferring from DTLK after instrument-free on TLK, which is inconsist with Yagi. Karikari et al. [(31)] reported osteoporotic patients were at risk of developing vertebral fractures and instrumentation failure, indicating it is imperative to optimize bone health with medical therapies such as vitamin D, calcium, bisphosphonates and parathyroid hormone.

The study firstly identifies the efficacy on DTLK improvement by only performing PLIF on LSS, which can proposal a new mentality on treatment DTLK and provide evidence for surgeon for preoperative conversation. The result puts a further explaination on the interaction between the focal deformity and whole spino-pelvic alignment. It also emphasizes the superiority of shorter-level fixation, supplementing and riching the theory of precision treatment. There were some limitations to be mentioned. Firstly, the sample in both groups is so small and a 
prospective, larger cohort with longer follow up will strength the conclusion. It can not provide surgical strategy for DTLK with LSS or stability in upper lumbar level (T12-L1 or L1-L2) where the instrument and fusion is inevitable. In addition, the results is not suitable for coronal deformity such as degenerative lumbar scoliosis or rigid TLK such as fracture-derived kyphosis, ankylosing spondylitis or Scheuermann's disease.

\section{Conclusions}

DTLK patients get improved by a mean of $8.9 \pm 11.5\left(^{\circ}\right)$ even if PLIF is only performed for LSS through a short-term follow up. TK and TLK both decreased at final compared to baseline. TLK and clinical parameters are comparable at last between L5 and S1 groups except a larger PT in S1 group. The clinical outcomes are indifferent in patients with corrected and uncorrected TLK. PI and PT can affect postoperative TLK and severe osteoporosis will impede TLK correction.

\section{List Of Abbreviations}

degenerative thoracolumbar kyphosis: DTLK

lumbar spinal stenois syndrome: LSS

lower instrumented vertebrae: LIV

the sacral slope: SS

pelvic tilt: $\mathrm{PT}$

pelvic incidence: $\mathrm{PI}$

upper instrumented vertebrea: UIV

posterior lumbar interbody fusion: PLIF

posterior-lateral fusion: PLF

thoracic kyphosis: TK

lumbar lordosis: LL

visual analogue scale: VAS

Oswestry disability index: ODI

\section{Declarations}

\section{- Ethics approval and consent to participate:}

We have acquired approval by local ethics committee (Ethics Committee of Peking University People's Hospital), the committee's reference number is not applicable. All authors have signed patient consent forms.

\section{- Consent for publication:}

Not Applicable

\section{- Availability of data and material:}

The data sets used and/or analyzed during the current study are available as an additional supporting files.

\section{- Competing interests:}

The authors declare that they have no competing interests.

\section{- Funding:}


National Key R\&D Program of China (grant number: 2016YFC0105606)

\section{- Authors' contributions:}

\section{- Conceptualization:}

LHY, MFQ; Data Curation: LHY, MFQ, BXG; Formal Analysis: BXG, XS, MFQ; Investigation: MFQ, BXG; Methodology: MFQ; XS, BXG; Project Administration: LHY; Resources: MFQ; BXG; Software: XS, LY; Validation: XS; Visualization: LHY; Writing \& Editing: LHY, MFQ, LY. All authors read and approved the final manuscript.

\section{- Acknowledgements:}

Houshan Lv.

\section{References}

1. Ahmed M, El-Makhy M, Grevitt M. The natural history of thoracolumbar kyphosis in achondroplasia. EUR SPINE J.2019 2019-1101;28(11):2602-7.

2. Lafian AM, Torralba KD. Lumbar Spinal Stenosis in Older Adults. Rheum Dis Clin North Am. 2018 2018-08-01;44(3):501-12.

3. Bayerl SH, Pohlmann F, Finger T, Franke J, Woitzik J, Vajkoczy P. The sagittal spinal profile type: a principal precondition for surgical decision making in patients with lumbar spinal stenosis. J Neurosurg Spine. 2017 2017-11-01;27(5):552-9.

4. Zeng Y, Qu X, Chen Z, Yang X, Guo Z, Qi Q, et al. Posterior corrective surgery for moderate to severe focal kyphosis in the thoracolumbar spine: 57 cases with minimum 3 years follow-up. EUR SPINE J. 2017 2017-07-01;26(7):1833-41.

5. Diebo BG, Shah NV, Stroud SG, Paulino CB, Schwab FJ, Lafage V. Realignment surgery in adult spinal deformity : Prevalence and risk factors for proximal junctional kyphosis. ORTHOPADE. 2018 2018-04-01;47(4):301-9.

6. Joukhadar N, Kubat O, Heflin J, Yasin MS, McClung A, Flynn T, et al. Superior Extension of Upper Instrumented Vertebrae in Distractionbased Surgery: A Surrogate for Clinically Significant Proximal Junctional Kyphosis. Spine Deform. 2019 2019-03-01;7(2):371-5.

7. Smith JS, Shaffrey Cl, Ames CP, Lenke LG. Treatment of adult thoracolumbar spinal deformity: past, present, and future. J Neurosurg Spine. 2019 2019-05-01;30(5):551-67.

8. Liang Y, Zhao Y, Wang T, Zhu Z, Liu H, Mao K. Precision Treatment of Adult Lumbar Degenerative Scoliosis Complicated by Lumbar Stenosis with the Use of Selective Nerve Root Block. WORLD NEUROSURG. 2018 2018-12-01;120:e970-5.

9. Ogura Y, Shinozaki Y, Kobayashi Y, Kitagawa T, Yonezawa Y, Takahashi Y, et al. Impact of decompression surgery without fusion for lumbar spinal stenosis on sagittal spinopelvic alignment: minimum 2-year follow-up. J Neurosurg Spine. 2019 2019-02-15:1-7.

10. Shin EK, Kim CH, Chung CK, Choi Y, Yim D, Jung W, et al. Sagittal imbalance in patients with lumbar spinal stenosis and outcomes after simple decompression surgery. SPINE J. 2017 2017-02-01;17(2):175-82.

11. Pierce KE, Horn SR, Jain D, Segreto FA, Bortz C, Vasquez-Montes D, et al. The Impact of Adult Thoracolumbar Spinal Deformities on Standing to Sitting Regional and Segmental Reciprocal Alignment. Int J Spine Surg. 2019 2019-08-01;13(4):308-16.

12. Klima S, Grunert R, Ondruschka B, Scholze M, Seidel T, Werner M, et al. Pelvic orthosis effects on posterior pelvis kinematics An in-vitro biomechanical study. Sci Rep. 2018 2018-10-29;8(1):15980.

13. Choi I, Jeon SR. The result of S1 double screw fixation in patients not suited for L4 and/or L5 pedicle screw insertion. Acta Neurochir (Wien). 2014 2014-02-01;156(2):229-33.

14. Dimai HP, Ljuhar R, Ljuhar D, Norman B, Nehrer S, Kurth A, et al. Assessing the effects of long-term osteoporosis treatment by using conventional spine radiographs: results from a pilot study in a sub-cohort of a large randomized controlled trial. SKELETAL RADIOL. 2019 2019-07-01;48(7):1023-32.

15. Lytsy $P$, cartographe $P$ in the right place: Revisiting the evidential value of P-values. 2018.

16. Goh TS, Shin JK, Youn MS, Lee HS, Kim TH, Lee JS. Surgical versus nonsurgical treatment of lumbar degenerative kyphosis. EUR SPINE J. 2017 2017-08-01;26(8):2153-9.

17. Zaina F, Tomkins-Lane C, Carragee E, Negrini S. Surgical versus non-surgical treatment for lumbar spinal stenosis. Cochrane Database Syst Rev. 2016 2016-01-29(1):D10264.

18. Redaelli A, Berjano P, Aebi M. Focal disorders of the spine with compensatory deformities: how to define them. EUR SPINE J. 2018 201802-01;27(Suppl 1):59-69. 
19. Bianco K, Norton R, Schwab F, Smith JS, Klineberg E, Obeid I, et al. Complications and intercenter variability of three-column osteotomies for spinal deformity surgery: a retrospective review of 423 patients. NEUROSURG FOCUS. 2014 2014-05-01;36(5):E18.

20. Ames CP, Scheer JK, Lafage V, Smith JS, Bess S, Berven SH, et al. Adult Spinal Deformity: Epidemiology, Health Impact, Evaluation, and Management. Spine Deform. 2016 2016-07-01;4(4):310-22.

21. Fujii K, Kawamura N, Ikegami M, Niitsuma G, Kunogi J. Radiological improvements in global sagittal alignment after lumbar decompression without fusion. Spine (Phila Pa 1976). 2015 2015-05-15;40(10):703-9.

22. Berjano P, Cecchinato R, Sinigaglia A, Damilano M, Ismael MF, Martini C, et al. Anterior column realignment from a lateral approach for the treatment of severe sagittal imbalance: a retrospective radiographic study. EUR SPINE J. 2015 2015-04-01;24 Suppl 3:433-8.

23. Lim JK, Kim SM. Comparison of Sagittal Spinopelvic Alignment between Lumbar Degenerative Spondylolisthesis and Degenerative Spinal Stenosis. J Korean Neurosurg Soc. 2014 2014-06-01;55(6):331-6.

24. Banno T, Arima H, Hasegawa T, Yamato Y, Togawa D, Yoshida G, et al. The Effect of Paravertebral Muscle on the Maintenance of Upright Posture in Patients With Adult Spinal Deformity. Spine Deform. 2019 2019-01-01;7(1):125-31.

25. Yasuda T, Hasegawa T, Yamato Y, Kobayashi S, Togawa D, Banno T, et al. Lumbosacral Junctional Failures After Long Spinal Fusion for Adult Spinal Deformity-Which Vertebra Is the Preferred Distal Instrumented Vertebra? Spine Deform. 2016 2016-09-01;4(5):378-84.

26. Hikata T, Watanabe K, Fujita N, Iwanami A, Hosogane N, Ishii K, et al. Impact of sagittal spinopelvic alignment on clinical outcomes after decompression surgery for lumbar spinal canal stenosis without coronal imbalance. J Neurosurg Spine. 2015 2015-10-01;23(4):451-8.

27. Miyakoshi N, Hongo M, Kobayashi T, Abe T, Abe E, Shimada Y. Improvement of spinal alignment and quality of life after corrective surgery for spinal kyphosis in patients with osteoporosis: a comparative study with non-operated patients. Osteoporos Int. 2015 2015-1101;26(11):2657-64.

28. Lafage V, Schwab F, Patel A, Hawkinson N, Farcy JP. Pelvic tilt and truncal inclination: two key radiographic parameters in the setting of adults with spinal deformity. Spine (Phila Pa 1976). 2009 2009-08-01;34(17):E599-606.

29. Barrey C, Roussouly P, Le Huec JC, D'Acunzi G, Perrin G. Compensatory mechanisms contributing to keep the sagittal balance of the spine. EUR SPINE J. 2013 2013-11-01;22 Suppl 6:S834-41.

30. Yagi M, Fujita N, Tsuji O, Nagoshi N, Asazuma T, Ishii K, et al. Low Bone-Mineral Density Is a Significant Risk for Proximal Junctional Failure After Surgical Correction of Adult Spinal Deformity: A Propensity Score-Matched Analysis. Spine (Phila Pa 1976). 2018 2018-0401;43(7):485-91.

31. Karikari IO, Metz LN. Preventing Pseudoarthrosis and Proximal Junctional Kyphosis: How to Deal with the Osteoporotic Spine. NEUROSURG CLIN N AM. 2018 2018-07-01;29(3):365-74.

\section{Figures}




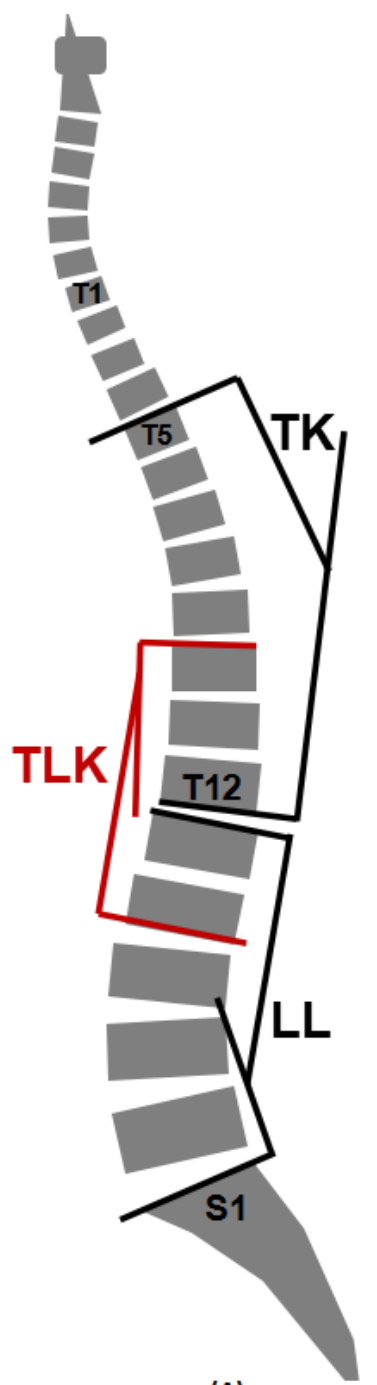

(A)

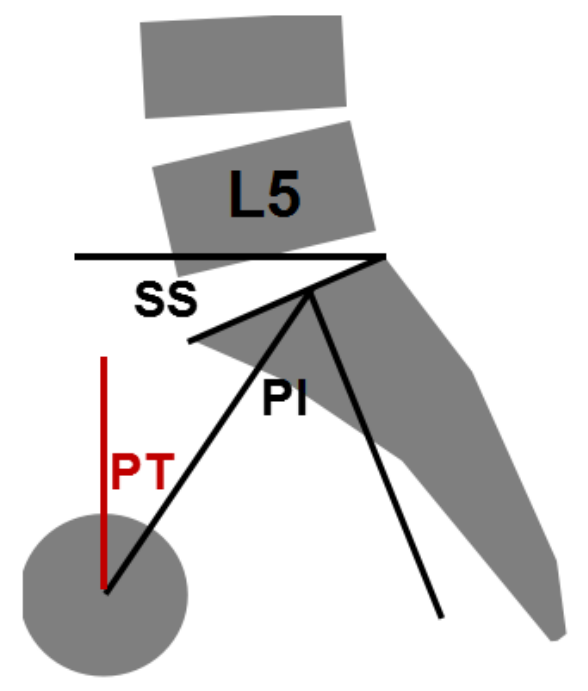

(B)

\section{Figure 1}

Diagram of spino-pelvic radiological parameters. (A) TK was the angle between upper endplate of T5 and lower endplate of T12. TLK was the angle between upper endplate of T10 and lower endplate of L2. LL was between upper endplate of L1 and upper endplate of S1. (B) PT was the angle between plumb line and the center of the femoral head to midpoint of upper endplate of S1. PI was the vertical line passing through the midpoint of upper endplate on S1, then second line connecting midpoint of the upper endplate on S1 and the femoral head and the angle between the second line and vertical line. SS was the angle between upper endplate of S1 and horizontal line.
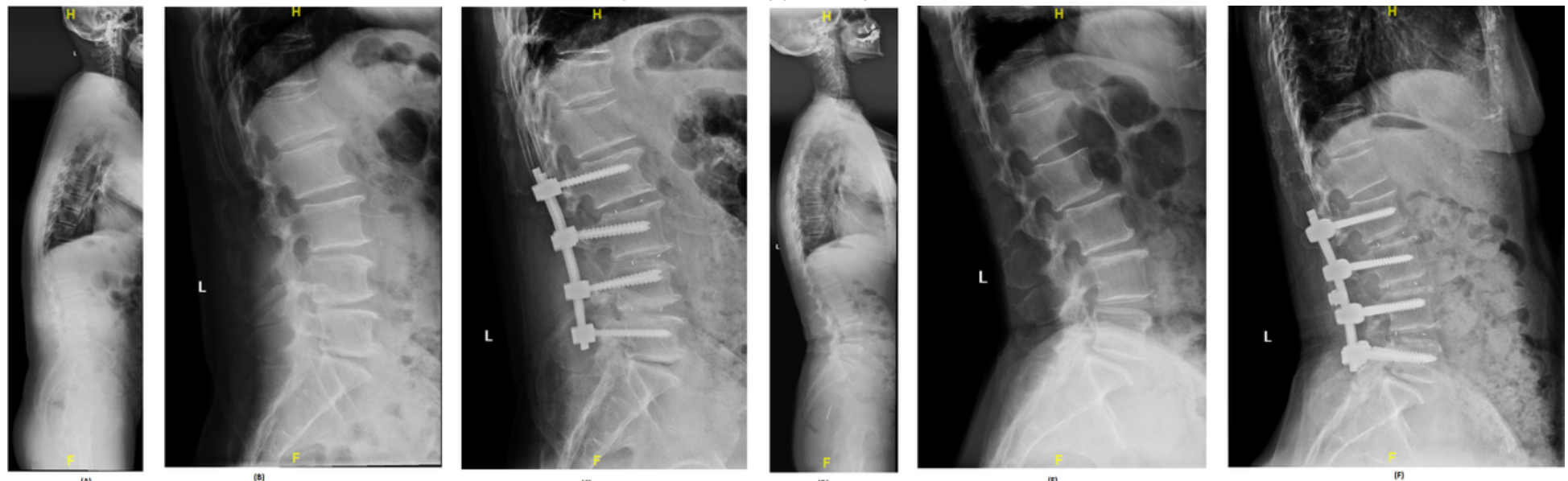
Figure 2

Cases on PLIF only for responsible levels with LSS, instability and disc hernia in DTLK patients. (A-C) 67 year-old male, he was performed L2L5 PLIF with a follow-up of 26 months. At baseline, TK=12.8, TLK=24.9, LL=17.8, PI=29.3, PT=4.2, SS=25.1, VAS=9 and ODI=44. At the endpoint, TK=10.5, TLK=16.7, LL=18.9, $\mathrm{PI}=29.0$, $\mathrm{PT}=6.6, \mathrm{SS}=22.4, \mathrm{VAS}=1$ and ODI=6. (D-F) 69 year-old female, she was performed L3-S1 PLIF with a follow-up of 23 months. At baseline, TK=33.9, TLK=20.4, LL=52.0, PI=44.4, PT=11.3, SS=33.1, VAS=6 and ODI=45. At the endpoint, $\mathrm{TK}=35.6, \mathrm{TLK}=9.8, \mathrm{LL}=47.9, \mathrm{PI}=43.6, \mathrm{PT}=15.4, \mathrm{SS}=28.2$, VAS=1 and ODI=6. Although there was postrior olisthesis of $\mathrm{L} 2$, L2-L3 kept in stability at the endpoint. 